\title{
Review
}

\section{Towards a Standard Vocabulary for Nanomaterials Relative to Regulatory Science}

\author{
Shuji Abe ${ }^{1}$ \\ National Institute of Advanced Industrial Science and Technology (AIST), Ibaraki, Japan \\ (Received December 24, 2010; Revised January 17, 2011; Accepted January 18, 2011)
}

\begin{abstract}
Substantial efforts have been made for the standardization of nanotechnology vocabulary by the ISO Technical Committee 229, which has already produced a few standards. However, in view of growing interest in the environmental, health, and safety issues of nanomaterials, the definition of nanomaterial still remains controversial in some respects, especially for regulatory purposes. This is related with difficulties in specifying requirements for a broad range of nanomaterials with a variety of applications.
\end{abstract}

Key words: nanotechnology, nanomaterial, terminology, standard

\section{Introduction}

A decade has passed since the announcement of the National Nanotechnology Initiative (NNI) by the USA President Clinton in 2000, which triggered world-wide competitions of nanotechnology research and development. The provisional definition of nanotechnology in the NNI was (1):

“. . research and technology development at the atomic, molecular, or macromolecular levels, in the length scale of approximately 1-100 nanometer range, to provide a fundamental understanding of phenomena and materials properties at the nanoscale and to model, create, characterize, manipulate, and use structures, devices, and systems that have novel properties and functions because of their small or intermediate size. The novel and differentiating properties and function are developed at a critical length scale of matter typically under 100 nanometers. Nanotechnology research and development includes integration of nanoscale structure into larger material components, systems, and architectures. Within these larger scale assemblies, the control and construction of their structures and components devices remain at the nanometer scale."

Initially, nanotechnology was considered a revolutionary technology with manipulation and control of matter at the level of atoms and molecules, as Drexler envisioned in his book "Engine of Creation"' (2). The futuristic vision of nanotechnology has lead to en- thusiasm as well as concern in the public. If a technology is revolutionary, its impact on the society must be strong. Some of the concerns were ethical questions about creating self-replicating molecular machines and about enhancing human abilities.

In an early report for the NNI anticipated that nanotechnology market will evolve in three phases: 1) Passive nanostructures (Year 2000-); 2) Active nanostructures (2005-); and 3) Systems of nanostructures (2010-) (3). However, this expectation turned out to be too optimistic. The actual progress has been rather slow, and the second and third categories are still in the stage of basic research. But we recognize that there has been a great deal of development in the first category, and we now have many products containing nanomaterials, which are well designed and engineered so as to have desired functions.

Owing to the slow development of nanotechnology, the initial ethical concern about nanotechnology has been mitigated, and the major concern has been more and more focused on the environmental, health, and safety (EHS) issues of nanomaterials. The number of toxicological studies on nanomaterials has increased rapidly worldwide. The OECD organized the Working Party on Manufactured Nanomaterials (WPMN) in 2006 to promote international collaborations on the risk assessment of nanomaterials. Although the risk evaluation of nanomaterials is still not easy, there is an increasing public pressure towards the regulation of nanomaterials. This poses a new demand for a practical definition of nanomaterials.

\section{Nanomaterials: New and Old}

The prefix "nano" simply means $10^{-9}$ in the SI unit system, so that $1 \mathrm{~nm}$ is $10^{-9} \mathrm{~m}$. The term "nanoscale" is commonly defined as size range from approximately 1 $\mathrm{nm}$ to $100 \mathrm{~nm}$ (4). In this sense, a nanomaterial is not a

${ }^{1}$ Correspondence to: Shuji Abe, Nanosystem Research Institute, National Institute of Advanced Industrial Science and Technology (AIST), Umezono 1-1-1, Tsukuba, Ibaraki 305-8568, Japan. Tel: +81-29-861-5370, Fax: +81-29-861-5375, E-mail: s.abe@aist.go.jp 
special material. Any chemical substance can have nanoscale structures therein. Many natural materials and biological materials have nanoscale structures. It would be difficult to find out materials without any nanoscale structures.

Actually, nanomaterials have been studied for a long time. An example is the research field of fine particles, which have diameters less than $10 \mu \mathrm{m}$, while ultrafine particles have diameters less than $100 \mathrm{~nm}$, i.e., in the nanoscale. The reason why the threshold for "ultrafine" is set at $100 \mathrm{~nm}$ is that this is roughly the resolution limit of optical microscope with the wavelength of visible light $400-800 \mathrm{~nm}$. Another example is colloid science. Colloids are mostly in the size range from $1 \mathrm{~nm}$ to $100 \mathrm{~nm}$ (or at most $1000 \mathrm{~nm}$ ). Because this size range is smaller than the wavelengths of light, colloid solutions are transparent, but can be observed by light scattering (Tyndall scattering).

There are many conventional products containing nanomaterials. For example, carbon black is an industrially manufactured colloidal carbon material in the form of spheres and of their aggregates with sizes below $1000 \mathrm{~nm}$. The size of a primary particle is typically between $5 \mathrm{~nm}$ and $50 \mathrm{~nm}$. Carbon black industry has a history of more than a century, and carbon black may be viewed as a material manufactured with a conventional technology. But it should be noted that many new products of carbon black is based on the precise control of its structure in the nanoscale.

Fine particles released from automobile engines were one of the major sources of air pollution. The term "particulate matter" (PM) has been used in this context. PM10 and PM2.5 implies particles whose aerodynamic diameter is less than $10 \mu \mathrm{m}$ and $2.5 \mu \mathrm{m}$, respectively. In recent years there has been an effort to reduce particulate matter smaller than $0.1 \mu \mathrm{m}$ (PM0.1 or ultrafine particle), such as diesel exhaust particles. This is in the category of incidental nanoparticles.

These "pre-nanotechnology" researches on ultrafine particles need to be distinguished from novel nanomaterials resulting from the application of various nanotechnologies. These "nanotechnology-based" nanomaterials are categorized as "manufactured nanomaterials", or "engineered nanomaterials" (see below).

\section{Definitions for Nanomaterials}

As mentioned in the previous section, the concept of nanomaterial is not very special, but rather covers a broad range of materials. Since a "nanomaterial" implies just a material in the nanoscale, it can be any material for any purpose. There are materials for cars and airplanes, materials for electronic devices, materials for houses, materials for food, materials for medicines, materials for cosmetics, etc. There are "hard" materials such as metal or semiconductor nanoparticles, carbon nanofibers and nanotubes. There are also "soft" materials such as nanocapsules for drug delivery systems or for food. In a sense, nanotechnology is a common concept or framework for all industries involving materials.

Thus it is obvious that there is a need for terminology standards. The ISO Technical Committee (TC) 229 "nanotechnologies" was established in 2005. One of the main tasks of this committee is to develop vocabulary standard for nanotechnologies, and it was assigned to Working Group (WG) 1 "Terminology and Nomenclature". The first project of the WG 1, proposed by the UK, was to develop a technical specification (TS) on terminology and definitions for nanoparticles. After a oneyear drafting, the document was published in 2008 as ISO/TS 27687 "Terminology and definitions for nanoobjects-Nanoparticle, nanofibre, and nanoplate".

The main question in this project was the definition of nanoparticle. It was discussed whether it should be limited to more or less spherical objects or should include objects with large aspect ratio, such as fibers and plates. It was finally decided that the term nanoparticle should be used for materials with all three dimensions in the nanoscale, distinct from nanofibers and nanoplates (4):

- nanoparticle: nano-object with all three external dimensions in the nanoscale

- nanofibre: nano-object with two similar external dimensions in the nanoscale and the third dimension significantly larger

- nanoplate: nano-object with one external dimension in the nanoscale and the two other external dimensions significantly larger

In these definitions "significantly larger" is interpreted as "more than three times larger". Then it was necessary to introduce an overarching term for these three concepts (4):

- nano-object: material with one, two or three external dimensions in the nanoscale

This term was newly invented by ISO but gained popularity rather quickly in the nanotechnology community.

The WG1 of ISO/TC229 has subsequently developing a series of vocabulary documents in collaboration with IEC (International Electrotechnical Commission)/TC 113 "Nanotechnology standardization for electrical and electronic products and systems":

- ISO/TS 80004-1 Nanotechnologies-Vocabulary-

Part 1: Core terms (published)

- ISO/TS 80004-2 Nanotechnologies-VocabularyPart 2: Nano-objects (planned as a revision of TS 27687)

- ISO/TS 80004-3 Nanotechnologies-VocabularyPart 3: Carbon nano-objects (published) 
- ISO/TS 80004-4 Nanotechnologies-VocabularyPart 4: Nanostructured materials

- ISO/TS 80004-5 Nanotechnologies-VocabularyPart 5: Nano/bio interface

- ISO/TS 80004-6 Nanotechnologies-VocabularyPart 6: Nanoscale measurement and instrumentation

- ISO/TS 80004-7 Nanotechnologies-Vocabulary-

Part 7: Diagnostics and therapeutics for healthcare

- ISO/TS 80004-8 Nanotechnologies-Vocabulary-

Part 8: Nanomanufacturing processes

It should be mentioned that Part 3 of the vocabulary series, published in April 2000, was developed with the lead of Japan. I myself was deeply involved in its development as the proposer and the project leader. It was very important to consult industries and academics in its development, since a definition must be both scientifically sound and practically acceptable.

Part 1 of the 80004 series was just published in October 2010. It deals with the term nanomaterial. Sometimes the term "nanomaterial" has been used to imply nano-objects. For example, the manufactured nanomaterials which the OECD/WPMN deals with were mostly nano-objects. But there are many other nanomaterials, such as composites made of nano-objects. Therefore, it was necessary to introduce a new term "nanostructured material" as a subcategory of nanomaterial. The two terms are defined (5) as:

- nanomaterial: material with any external dimension in the nanoscale or having internal structure or surface structure in the nanoscale

- nanostructured material: material having internal nanostructure or surface nanostructure

Here, a "nanostructure" implies a composition of interrelated constituent parts in which one or more of those parts is a nanoscale region. The definition of nanostructured material includes aggregates and agglomerates of nano-objects. Nanomaterial is an overarching term for both nano-objects and nanostructured materials. Note that a nano-object can be a nanostructured material at the same time if it has an internal or surface nanostructure. In this case it is recommended to call it a nano-object, reserving the term "nanostructured material" mainly for materials with external dimensions larger than $100 \mathrm{~nm}$. Figure 1 shows the hierarchy of nanomaterial.

For regulatory purposes, it is important to clarify distinction between natural nanomaterials, incidental, and manufactured or engineered nanomaterials, as defined in ISO/TS 80004-1 (5) as:

- incidental nanomaterial: nanomaterial generated as an unintentional by-product of a process

- engineered nanomaterial: nanomaterial designed for specific purpose or function

- manufactured nanomaterial: nanomaterial intentionally produced for commercial purpose to have specific

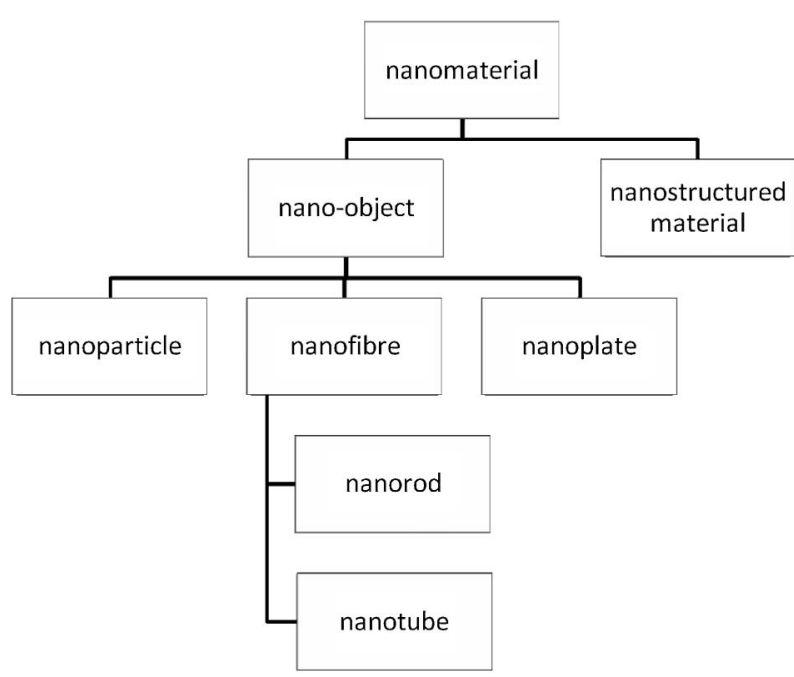

Fig. 1. Hierarchy of nanomaterial terms defined by ISO $(4,5)$.

properties or specific composition

There is a subtle difference between 'engineered' and 'manufactured'. The same adjectives apply for all the subcategories of nanomaterial, such as "manufactured nano-objects"'.

\section{Nanomaterials and Regulation}

Recently the definition of nanomaterial for the regulatory purpose has become a critical issue. There have been a handful of definitions of nanomaterial proposed by various organizations (6). A good summary of proposed definitions was given by Lövestam et al. (7). For example, the EU is introducing a regulation of nanomaterials in cosmetic products (8), where nanomaterial is defined as "an insoluble or biopersistent and intentionally manufactured material with one or more external dimensions, or an internal structure, on the scale from 1 to $100 \mathrm{~nm}$ '. An immediate question is what is exactly meant by 'insoluble' and 'biopersistent'.

The Scientific Committee on Emerging and Newly Identified Health Risks (SCENIHR) of the European Commission (EC) released a document entitled Scientific Basis for the Definition of the Term "Nanomaterial" for public consultation (9). Subsequently, the EC released a draft Recommendation on the Definition of the Term "Nanomaterial" (10).

A new element in these definitions is the consideration of the size distribution. The proposed EC definition reads:

Nanomaterial: means a material that meets at least one of the following criteria:

- consists of particles, with one or more external dimensions in the size range $1 \mathrm{~nm}-100 \mathrm{~nm}$ for more than $1 \%$ of their number size distribution;

- has internal or surface structures in one or more dimensions in the size range $1 \mathrm{~nm}-100 \mathrm{~nm}$; 
- has a specific surface area by volume greater than $60 \mathrm{~m}^{2} / \mathrm{cm}^{3}$, excluding materials consisting of particles with a size lower than $1 \mathrm{~nm}$.

Among the three criteria, the first and the third criteria refer to nano-objects. The condition of "more than $1 \%$ of their number size distribution" means that we must measure or estimate the entire number size distribution of particles in the sample by some method. This is quite a challenging requirement. The threshold value, $1 \%$ in this case, is also in question. Is it too small? Is it reasonable to use the number distribution rather than the weight distribution? These questions need to be discussed carefully among various stakeholders before reaching national or international consensus.

The Australian government is introducing new regulatory framework where industrial nanomaterials are considered to be new chemicals (11). Their working definition of "industrial nanomaterial" is "intentionally produced, manufactured or engineered to have unique properties or specific composition at the nanoscale, that is a size range typically between $1 \mathrm{~nm}$ and $100 \mathrm{~nm}$, and is either a nano-object or is nanostructured". It is stated that 'unique properties' refers to "chemical and/or physical properties that are different because of its nanoscale features as compared to the same material without nanoscale features, and result in unique phenomena (e.g., increased strength, chemical reactivity or conductivity) that enable novel applications"'.

This specification of unique properties is an important aspect of nanotechnology, as mentioned in Introduction. Here I would like to refer to the definition of nanotechnology given by ISO (5):

- nanotechnology: the application of scientific knowledge to manipulate and control matter in the nanoscale to make use of size- and structure-dependent properties and phenomena distinct from those associated with individual atoms or molecules or with bulk materials

We note that the 'distinct' properties are the purpose of nanotechnology. When nanomaterials are used in products, they are used for a specific purpose or for a specific benefit. In this sense, a nanomaterial is not just a 'nanoscale' material (characterized by size only) but rather a 'nanotech' material (characterized by size and properties). Further deliberation will be necessary about how to define and characterize the functional aspect of nanomaterials more appropriately.

This problem is also related with the labelling of 'nano' products. Right now the European Commission for standardization (CEN) is developing "Guidance on the labeling of manufactured nano-objects and products containing manufactured nano-objects" jointly with the ISO/TC 229. This was proposed by the UK on the basis of BS PAS 130 (12). It is necessary to have a common understanding among manufacturers (throughout supply chains), retailers and consumers about what is really 'nano' inside the products. This is an important effort towards the responsible development of nanotechnology beneficial to the society.

Acknowledgment: The author wishes to thank Dr. Fred Klaessig and Dr. Takuya Igarashi for useful discussions.

\section{References}

1 US National Research Council, Division on Engineering and Physical Sciences. Small wonders, endless frontiers-A review of the national nanotechnology initiative. Washington D.C.: National Academy Press, 2002.

2 Drexler KE. Engines of creation: The coming era of nanotechnology, New York: Random House; 1986.

3 Roco MC. The national nanotechnology initiative: past, present, future. In: Goddard WAIII, Brenner D, Lyshevski SE, Iafrate GJ, editors. Handbook of nanoscience, engineering, and technology. 2nd ed. CRC Press; 2007. p. 3-1-3-26.

4 ISO/TS 27687: 2008 Nanotechnologies-Terminology and definitions for nano-objects-nanoparticle, nanofibre, and nanoplate.

5 ISO/TS 80004-1: 2010 Nanotechnologies-VocabularyPart 1: Core terms.

6 Klaessig F, Marrapese M, Abe S. Current perspectives in nanotechnology terminology and nomenclature. In: Murashov V, Howard J, editors. Nanotechnology standards. Springer; in press.

7 Lövestam G, Rauscher H, Roebben G, Klüttgen BS, Gibson N, Putaud JP, Stamm H. Consideration on a definition of nanomaterial for regulatory purposes. EC, Joint Research Centre, 2010.

8 EU Cosmetics Product Regulation 1223/2009.

9 SCENIHR (Scientific Committee on Emerging and Newly Identified Health Risks), EU. Scientific basis for the definition of the term "nanomaterial", Pre-consultation opinion. 6 July 2010.

10 EC (European Commission). Draft recommendation: on the definition of the term "nanomaterial". October 2010.

11 NICNAS (National Industrial Chemicals Notification and Assessment Scheme). Chemical Gazette, No. C10. Australian Government, 5 October 2010.

12 BS PAS 130: 2007 Guidance on the labeling of manufactured nanoparticles and products containing manufactured nanoparticles. 